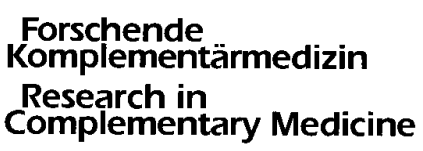

\section{Annual Symposium on Complementary Health Care}

This annual scientific gathering has fast become a 'state of the art' meeting of international acclaim. There are, of course, many conferences on complementary medicine but this one is different in several ways. In addition to being a regular event with an emphasis on international contributions, its format is that of rigorous research symposium which so far has not been adopted by complementary medicine. Abstracts are invited for the deadline of 1 September each year. These submissions are then reviewed by a panel of experts. Only those abstracts which contain original data and are of high quality are chosen. The submitted abstracts are then assembled under subject areas which eventually represent the sessions at the meeting. In addition several keynote lecturers are invited to give topical overviews, and the Annual Award in Complementary Medicine is presented to each year's prize winner who also gives the award lecture. Finally there are workshops to supplement the programme.

This year the spectrum of topics included research methodology, phytomedicine, herbalism, acupuncture, homoeopathy, safety and cancer. There were more than 60 oral presentations from delegates from 14 different countries. A crucial part of the meeting were the discussions, and the organisers insisted that there was enough time for them. The discussions were often intentionally critical but they remained open, factual and constructive at alle times.

The aim for the future is not so much to increase the size of the meeting but to continue ameliorating its quality. Considering its humble beginnings 4 years ago, the Annual Symposium on Complementary Health Care has come a long way. There can be little doubt that it has become one of the most important conferences on complementary medicine today. The next symposium will take place 10-12 December 1998. Enquiries to symposium secretary tel./fax +44 1392424989 at this Department.

E. Ernst, Exeter

Das Annual Symposium on Complementary Health Care, initiiert und organisiert vom Department of Complementary Medicine der Universität Exeter, fand vom 10. bis 12. Dezember 1997 im englischen Exeter statt. Das Symposium, das bereits zum vierten Male veranstaltet wurde, zog wie in den vergangenen Jahren eine beachtliche Zahl Interessierter aus allen Bereichen der Gesundheitsversorgung an. Etwa 140 Wissenschaftler und Beobachter aus sechs europäischen Ländern sowie USA, Kanada, China und Indien waren anwesend und präsentierten in mehr als 60 Vorträgen ihre Daten. Präsentiert wurden hauptsächlich Originalarbeiten mit zum Teil herausragender Qualität.
Auf erneute Anfrage wurde das Symposium wieder mit einem Workshop eröffnet. Unter dem Vorsitz von Prof. Edzard Ernst wurden methodologische Fragestellungen an konkreten Fallbeispielen erörtert und Lösungsmöglichkeiten diskutiert.

Die erste Hauptvorlesung wurde von Dr. David Reilly vom Glasgow Homoeopathic Hospital gehalten, der über die therapeutische Bedeutung der ärztlichen Konsultation referierte und diese klar als einen der Eckpfeiler der Therapie identifizierte. Weitere Hauptvorlesungen wurden von Ted Kaptchuk von der Harvard Medical School sowie von Dr. Peter Fisher vom Royal London Homoeopathic Hospital gehalten; letzterer sprach über essentielle methodologische Aspekte und beleuchtete diese mit Blick auf komplementärmedizinische Fragestellungen.

Einzelne Vortragsblöcke waren ausschliesslich Themengebieten wie der Homöopathie oder Nebenwirkungen komplementärmedizinischer Therapiemassnahmen gewidmet. Andere spiegelten die ganze Vielfalt der Komplementärmedizin wider und beinhalteten Vorträge zur Akupunktur und manuellen Medizin sowie Beiträge zur Fussreflexzonenzmassage, Ernährung, Aromatherapie und Shiatsu. Phytomedizin war wieder ein Schwerpunkt dieser zweieinhalb Tage dauernden Konferenz. Unter anderem wurden neueste Ergebnisse zur Bioverfügbarkeit von $\beta$-Aescin, dem Wirkstoff der Rosskastanie, sowie Daten zur Wirksamkeit von Artischockenextrakt bei dyspeptischem Syndrom vorgestellt.

Ein Höhepunkt der Veranstaltung war sicherlich die Vergabe des diesjährigen Forschungspreises an Prof. David Overstreet vom Skipper Bowels Center for Alcohol Studies der Universität North Carolina. In seinem Festvortrag zeichnete er die Entwicklung eines traditionellen Heilmittels zum laborchemisch erprobten Pflanzenextrakt mit Wirkungen bei Alkoholabhängigkeit nach.

Das diesjährige Symposium übertraf an Qualität der präsentierten Arbeiten sowie an Themenvielfalt das der vergangenen Jahre. Mit seinem Anspruch an hochwertige Forschung scheint es sich als Forum auf dem Gebiet der Komplementärmedizin etabliert zu haben. Die Konferenzabstracts sind in der Dezember-Ausgabe von FACT [1] veröffentlicht. Informationen zu FACT sowie zum nächstjährigen Symposium, das vom 10. bis 12. Dezember 1998 wieder in Exeter stattfinden wird, sind zu beziehen über Mrs. Elizabeth Stewart, Department of Complementary Medicine, Postgraduate Medical School, University of Exeter, 25 Victoria Park Road, Exeter EX2 4NT. Tel. und Fax: +441392424989.

M. H. Pittler, Exeter

\section{Literatur}

1 4th Annual Symposium on Complementary Health Care, 10th-12th December 1997, Exeter, UK. FACT 1997;2:184-199. 